\title{
Carbapenem-Resistant Bacteria and Laboratory Detection Methods
}

\author{
Mohammad Rahmati Roodsari ${ }^{1}$, Fatemeh Fallah ${ }^{2}$, Arezou Taherpour ${ }^{3}$, Mojdeh Hakemi \\ Vala $^{4}$, Ali Hashemi ${ }^{4, *}$ \\ ${ }^{1}$ Shahid Beheshti University of Medical Sciences, Tehran, IR Iran \\ 2 Pediatric Infections Research Center, Shahid Beheshti University of Medical Sciences, Tehran, IR Iran \\ 3 Pepartment of Microbiology, Kurdistan University of Medical Sciences, Sanandaj, IR Iran \\ ${ }^{4}$ Department of Microbiology, Kurdistan University of Medical Sciences, Sanandaj, IR Iran \\ *Corresponding author: Ali Hashemi, Department of Microbiology, Shahid Beheshti University of Medical Sciences, P. O. Box: 19857-17443, Tehran, IR Iran. Tel: +98-2123872556, Fax: \\ +98-2144503530, E-mail: ali.hashemi@sbmu.ac.ir.
}

Received: April 28, 2012; Revised: January 01, 2013; Accepted: July 02, 2013

Carbapenemase producing bacteria frequently possess resistance mechanisms to a wide-range of antibacterial agents, and are associated with high mortality and morbidity rates. Some enzymes like extended-spectrum $\beta$-lactamase (ESBL), New Delhi metallobeta-lactamase-1 (NDM-1), Klebsiella pneumoniae carbapenemase (KPC), and OXA-producing Enterobacteriaceae are very important in frequently isolated nosocomial bacteria, which cause resistant to many classes of drugs. NDM-1 or KPCs, is a worldwide health problem which is slowly increasing. There is no vaccine to prevent infections produced by carbapenem-resistant bacteria.

Keywords: Beta-lactamase NDM-1; Plasmids; Drug Resistance, Microbial

\section{Introduction}

\subsection{Emergence of Carbapenem Resistance}

Carbapenemases, in gram-negative bacterial clinical strains are an increasing concern because they confer resistance to all beta-lactam antibiotics. The problem of dissemination of carbapenemases in several Mediterranean countries was observed mainly in $P$. aeruginosa. Later on, the most common carbapenemase in Europe among Enterobacteriaceae was Verona integron-encoded metallo- $\beta$ lactamase (VIM) among K. pneumoniae and K. pneumoniae carbapenemase (KPC). Other particularly problematic carbapenemases are the New Delhi metallo- $\beta$-lactamase (NDM), which is highly prevalent in the Indian subcontinent and the Middle East. The OXA-48-like enzymes have caused outbreaks in several European countries and are now spreading rapidly (1). Resistance to carbapenems has recently emerged in Enterobacteriaceae and has resulted in serious clinical consequences as carbapenems are practically the last option to treat infections due to these bacteria. The Ambler classes A, B, and D $\beta$-lactamases are classes of carbapenemases in Enterobacteriaceae. There are various classes of A carbapenemases: some of them are chromosome-associated (NmcA, Sme, IMI-1, SFC-1), and others are encoded by plasmid (Klebsiella pneumoniae carbapenemases [KPC], IMI-2, GES, derivatives). Anyway, Carbapenems are hydrolyzed by all of them and Clavulanic acid can partially inhibit the effect of these enzymes (2). Class B metallo- $\beta$-lactamases (MBLs) are mostly encoded by VIM and IMP types. In addition, the New Delhi metallo$\beta$-lactamase-1 (NDM-1) has been added as a new metallo$\beta$-lactamase type. In 1991, acquisition MBL and IMP-1 in Serratia marcescens had been reported for the first time. After that time MBLs have been reported worldwide. All $\beta$-lactams are hydrolyzing by these enzymes, exceptionally Aztreonam. Ethylenediaminetetraacetic acid (EDTA) can inhibit their activity but Clavulanic acid cannot do that $(2,3)$. Class D types are most encoded by OXA-48 and OXA-181. Now, OXA-48 producers distribute in Europe, in the southern and eastern part of the Mediterranean sea, and Africa. But they have not been reported from the United States and Canada. OXA-48 producers were isolated from hospitalized patients who were hospitalized in endemic source. OXA-181 a point mutant analog of OXA-48 has been isolated from India or Indian origin. Carbapenems and broad-spectrum Cephalosporins, such as Ceftazidime, and Aztreonam are weakly hydrolyzed by OXA-48/OXA-18. EDTA or Clavulanic acid (resistance to Amoxicillin/Clavulanic acid) cannot inhibit their activ-

Implication for health policy/practice/research/medical education:

As remarkable results; the importance of these enzymes such as NDM-1 and KPC are comparable with HIV, tuberculosis and malaria. There is no vaccine to prevent infections produced by bacteria which have carbapenemases, yet. So, it is necessary to inspect phenotyping and genotyping NDM-1 resistant pattern world-wide.

Copyright @ 2014, Pediartric Infections Research Center. This is an Open Access article distributed under the terms of the Creative Commons Attribution License (http://creativecommons.org/licenses/by/3.0), which permits unrestricted use, distribution, and reproduction in any medium, provided the original work is properly cited. 
ity. OXA-48 producers are most in K. pneumoniae and E. coli. Identification of OXA-48-type producers is difficult. Therefore, true prevalence estimation is difficult too $(4,5)$.

\section{What is NDM-1?}

Some bacteria carry a gene (DNA code) named NDM-1, which stands for New Delhi metallo-beta-lactamase-1. NDM-1 producing bacteria are resistant to nearly all antibiotics, including carbapenem antibiotics which are also known as antibiotics of the last resort. Because, NDM-1 gene makes the bacterium produce an enzyme which neutralizes the activity of carbapenem antibiotics. These bacteria are the most powerful superbug around. Such bacteria are usually susceptible only to "Polymyxin" polymyxins and "Tigecycline" tigecycline. Recently, emergence of NDM-1 enzymes is considered as a major threat, because bacteria which possess this beta-lactamase are resistant to almost all $\beta$-lactam drugs $(6,7)$ like Fluoroquinolones and Aminoglycosides (8), except Aztreonam $(7,9)$, Tigecycline and Colistin. However, bacteria could be resistant to the last three drugs (10) and also Fosfomycin (11). In 2009, the NDM-1 enzyme was reported for the first time. It was detected in a Swedish patient who had traveled to India and was hospitalized in New Delhi during 2007. He acquired urinary tract infection (UTI) due to carbapenem resistant Klebsiella pneumoniae (12). Enterobacteriaceae NDM-1 producers cause a wide range of infections such as septicemia, UTI, diarrhea, pulmonary infections, peritonitis, devices- associated and soft tissue infections (9). The bacteria with NDM-1 enzyme are known as superbugs and public health must pay more attention to them (13), and centers for disease control and prevention (CDC) have guidelines to control strains with NDM-1 (6). The most common types of infections with NDM-1 producer's bacteria have been reported in adults, but blaNDM-1 in two strain of K. pneumonia was reported from a neonatal intensive care unit (NICU) in India (14). More than 6 different NDM allotypes are known (Table 1).

Table 1. NDM-type $\beta$-Lactamases (Reprinted from www.lahey. org/studies/).

\begin{tabular}{lll}
\hline Enzyme & Nucleotide & Reference \\
\hline NDM-1 & FN396876 & AAC 53:5046-5054, 2009 \\
NDM-2 & JF703135 & JAC 66:1260-1262, 2011 \\
NDM-3 & Assigned & \\
NDM-4 & Assigned & \\
NDM-5 & JN104597 & \\
NDM-6 & JN967644 & \\
\hline
\end{tabular}

The NDM-1 gene is carried on a plasmid or chromosome. The rapid emergence of the NDM-1 gene has been related to movable plasmids which can move among different bacteria, subsequently, this gene can spread throughout the world (7). Plasmids with the NDM-1 gene can be trans- mitted to other bacteria, even to the human intestinal flora. There are about 10 - 100 trillion bacteria as human normal flora which can be causative of many problems in human. It has been estimated that about 100 million Indian carry bacteria with NDM-1 gene as normal gut flora (15). The blaNDM-1 gene is located on different plasmids and can harbor a large number of resistance genes. These genes are carbapenemase genes (OXA 48, OXA 181 and VIM). Aminoglycoside resistance genes (16s rRNA methylase), plasmid- associated cephalosporinase genes (CMY16, CMY- 58), class A genes (KPC), ESBL genes (TEM,CTX-M15,SHV-12), macrolides resistance genes (esterase), qnr genes (qnrAB, qnr B1, qnr B2) and Rifampin resistance gene. The above-mentioned plasmids (with blaNDM-1) do as multidrug resistance (MDR) pools and cause pan-drug resistance $(16,17)$. A paper in lancet infectious disease journal (2010) showed that NDM-1 gene cases had been isolated from India, Pakistan and the UK by molecular, epidemiological and biological studies (12). About 70 cases have been identified in the UK, 150 in India and Pakistan, 4 in Australia, 20 in Austria, 2 in Belgium, 4 in Canada, 3 in China, 1 in Germany, 1 in Hong Kong, 1 in Singapore, 1 in Sweden and cases in Taiwan, America, France, Kenya and Italia were 1, 3, 2, 7 and 1 case (s), respectively (9). Recently, CDC has suggested NDM- 1 as a transmissible agent, especially in patients who have been hospitalized in Pakistan and India. There are NDM-1 pathogens in acquired infections from hospital environment and community (12).

\section{How Untreatable Is NDM-1 Producing Bacteria?}

NDM-1 is spreading in India and Pakistan. It has reached Europe, the USA, Canada and Australia. NDM1 producing bacterial strains are resistant to all antibiotics. Physicians in the UK have managed to fight these infections with a combination of several different drugs. Currently, surveillance, prompt identification, isolation of infected patients, disinfecting hospital equipment, and thorough hand-hygiene procedures in hospitals are the only ways to combat the spread of NDM-1. This is going to be a challenge and will require international cooperation (9).

\section{How Is NDM-1 Detected?}

Some pathogens with NDM-1 and KPC may not be recognized by common laboratory tests; consequently, unaffected antibiotics are prescribed in the wrong way, therefore causes emergence and spread of more resistant bacteria $(15,18)$. The E- test strips are recommended to identify MBL producing bacteria; it is based on metallo$\beta$-lactamases inhibition by EDTA. These strips have two parts Imipenem and Imipenem-EDTA. The E- test MBL is reliable for detection of resistance, except in Enterobacter cloacae and K. pneumoniae (19). It can be substituted by DDST after applying an Imipenem disc $10 \mathrm{~mm}$ apart from a disc containing approximately $1900 \mathrm{mg}$ of EDTA. If Imi- 
penem-EDTA disc inhibition zone is more than $4 \mathrm{~mm}$ and the inhibition zone is not seen for Imipenem disc, strain is considered as metallo- $\beta$-lactamase positive (such as NDM-1). The Hodge-test has low specificity, therefore, it is not recommended for metallo- $\beta$-lactamase identification (20). Carbapenemase production is detected by the Modified Hodge test (MHT) when the test isolate produces the enzyme and allows the growth of a carbapenem susceptible strain (E. coli ATCC 25922) towards a Carbapenem disk. The result is a characteristic cloverleaf-like indentation. (21). Except class D carbapenemases, Meropenem associated inhibitor is very specific and sensitive to detect carbapenemases. The microarray is a very precise method to detect multidrug Enterobacteriaceae $\beta$-lactamase positive (22). Also, Real time-PCR method is a useful technique. Advantages of this method are specificity and sensitivity, rapid detection of NDM-1 producers in less than 2 hours; also it can be used to detect KPC, OXA, VIM and IMP-type (23). The other technique; Loop-mediated isothermal amplification (LAMP) was used to detect NDM-1 producers in 2011 (24). To screen carbapenemases such as NDM and KPC enzymes, the rapid multiplex- PCR (less than 4 hours) is reliable (25). Pulsed-filed gel electrophoresis (PFGE) and multilocus sequence typing (MLST) are two techniques to determine the identity and genotype of $\beta$-lactamase-related plasmid genes like $\operatorname{NDM}(7,26,27)$. So far, in Iranian studies on P. aeruginosa, the emphasis was on identification of Ambler class A and Ambler class D serine OXA and also three reports on Ambler class B betalactamases (27-32). As remarkable results; NDM-1 or KPCs, is a worldwide health problem which is slowly becoming as important as HIV infection, extremely drug-resistant tuberculosis and malaria. There is no vaccine to prevent infections produced by Carbapenem-resistant bacteria. So, it is necessary to inspect phenotyping and genotyping of NDM-1 and KPC resistant pattern world-wide.

\section{Acknowledgements}

With all due respects, this review is presented to researchers who work on drug-resistant bacteria.

\section{Authors' Contribution:}

All the authors contributed in writing this article.

\section{Financial Disclosure:}

The authors declare no competing financial interests.

\section{Funding/Support:}

There is no support for this study.

\section{References}

1. Giske CG, Martinez-Martinez L, Cantón R, Stefani S, Skov R, Glupczynski Y, Nordmann P, Wootton M, Miriagou V, Simonsen GS, Zemlickova H, Cohen-Stuart J, Gniadkowski M. EUCAST; 2012. [Decem- ber]. EUCAST guidelines for detection of resistance mechanisms and specific resistances of clinical and/or epidemiological importance. Available from: https:// www.eucast.org.

2. Queenan AM, Bush K. Carbapenemases: the versatile beta-lactamases. Clin Microbiol Rev. 2007;20(3):440-58.

3. Walsh TR, Toleman MA, Poirel L, Nordmann P. Metallo-betalactamases: the quiet before the storm? Clin Microbiol Rev 2005;18(2):306-25.

4. Carrer A, Poirel L, Yilmaz M, Akan OA, Feriha C, Cuzon G, et al. Spread of OXA-48-encoding plasmid in Turkey and beyond. Antimicrob Agents Chemother. 2010;54(3):1369-73.

5. Poirel L, Ros A, Carrer A, Fortineau N, Carricajo A, Berthelot P, et al. Cross-border transmission of OXA-48-producing Enterobacter cloacae from Morocco to France. J Antimicrob Chemother: 2011;66(5):1181-2.

6. Jean SS, Hsueh PR. High burden of antimicrobial resistance in Asia. Int J Antimicrob Agents. 2011;37(4):291-5.

7. Roy S, Singh AK, Viswanathan R, Nandy RK, Basu S. Transmission of imipenem resistance determinants during the course of an outbreak of NDM-1 Escherichia coli in a sick newborn care unit. J Antimicrob Chemother. 2011;66(12):2773-80.

8. Rogers BA, Aminzadeh Z, Hayashi Y, Paterson DL. Country-tocountry transfer of patients and the risk of multi-resistant bacterial infection. Clin Infect Dis. 2011;53(1):49-56.

9. Nordmann P, Poirel L, Toleman MA, Walsh TR. Does broad-spectrum beta-lactam resistance due to NDM-1 herald the end of the antibiotic era for treatment of infections caused by Gram-negative bacteria? J Antimicrob Chemother. 2011;66(4):689-92.

10. Pfeifer Y, Wilharm G, Zander E, Wichelhaus TA, Gottig S, Hunfeld $\mathrm{KP}$, et al. Molecular characterization of blaNDM-1 in an Acinetobacter baumannii strain isolated in Germany in 2007. J Antimicrob Chemother. 2011;66(9):1998-2001.

11. Yang J, Ye L, Wang W, Luo Y, Zhang Y, Han L. Diverse prevalence of $16 \mathrm{~S}$ rRNA methylase genes armA and rmtB amongst clinical multidrug-resistant Escherichia coli and Klebsiella pneumoniae isolates. Int J Antimicrob Agents. 2011;38(4):348-51.

12. Arya SC, Agarwal N. International travel with acquisition of multi-drug resistant Gram negative bacteria containing the New Delhi metallo-beta-lactamase gene, bla NDM-1. Travel Med Infect Dis. 2011;9(1):47-8.

13. Bonomo RA. New Delhi metallo-beta-lactamase and multidrug resistance: a global SOS? Clin Infect Dis. 2011;52(4):485-7.

14. Roy S, Viswanathan R, Singh AK, Das P, Basu S. Sepsis in neonates due to imipenem-resistant Klebsiella pneumoniae producing NDM-1 in India. J Antimicrob Chemother. 2011;66(6):1411-3.

15. Walsh TR, Toleman MA. The emergence of pan-resistant Gramnegative pathogens merits a rapid global political response. $J$ Antimicrob Chemother. 2012;67(1):1-3.

16. Lascols C, Hackel M, Marshall SH, Hujer AM, Bouchillon S, Badal R, et al. Increasing prevalence and dissemination of NDM-1 metallobeta-lactamase in India: data from the SMART study (2009). J Antimicrob Chemother. 2011;66(9):1992-7.

17. Bogaerts P, Bouchahrouf W, de Castro RR, Deplano A, Berhin C Pierard D, et al. Emergence of NDM-1-producing Enterobacteriaceae in Belgium. Antimicrob Agents Chemother. 2011;55(6):3036-8.

18. Landman D, Bratu S, Quale J. Contribution of OmpK36 to carbapenem susceptibility in KPC-producing Klebsiella pneumoniae. JMed Microbiol. 2009;58(Pt 10):1303-8.

19. Shakibaie MR, Shahcheraghi F, Hashemi A, Adeli NS. Detection of TEM, SHV and PER Type Extended-Spectrum ß-Lactamase Genes among Clinical Strains of Pseudomonas aeruginosa Isolated from Burnt Patients at Shafa-Hospital, Kerman, Iran. Iran J Basic Med Sci. 2008;2(11):104-11.

20. Nordmann P, Poirel L, Carrer A, Toleman MA, Walsh TR. How to detect NDM-1 producers. J Clin Microbiol. 2011;49(2):718-21.

21. Castanheira M, Deshpande LM, Mathai D, Bell JM, Jones RN Mendes RE. Early dissemination of NDM-1- and OXA-181-producing Enterobacteriaceae in Indian hospitals: report from the SENTRY Antimicrobial Surveillance Program, 2006-2007. Antimicrob Agents Chemother. 2011;55(3):1274-8.

22. Naas T, Cuzon G, Bogaerts P, Glupczynski Y, Nordmann P. Evalua- 
tion of a DNA microarray (Check-MDR CT102) for rapid detection of TEM, SHV, and CTX-M extended-spectrum beta-lactamases and of KPC, OXA-48, VIM, IMP, and NDM-1 carbapenemases. J Clin Microbiol. 2011;49(4):1608-13.

23. Ong DC, Koh TH, Syahidah N, Krishnan P, Tan TY. Rapid detection of the blaNDM-1 gene by real-time PCR. J Antimicrob Chemother. 2011;66(7):1647-9.

24. Zhang Y, Wu N, Zhu B, Chen L, Zhu Y. [Establishment of loop-mediated isothermal amplification technique for rapid detection of NDM-1 gene]. Sheng Wu Gong Cheng Xue Bao. 2011;27(8):1232-8.

25. Kaase M, Nordmann P, Wichelhaus TA, Gatermann SG, Bonnin RA, Poirel L. NDM-2 carbapenemase in Acinetobacter baumannii from Egypt. J Antimicrob Chemother. 2011;66(6):1260-2.

26. Fallah F, Taherpour A, Hakemi Vala M, Hashemi A. Global spread of New Delhi metallo-beta-lactamase-1(NDM-1). Iran J Clin Infect Dis. 2011;6(4):171-77.

27. Fallah F, Hakemi Vala M, Hashemi A, Shams S. Emergence of Novel Plasmid-mediated Beta-lactamase in Klebsiella pneumonia. Qom Univ Med SciJ. 2013;6(4):104-16.
28. Bahar MA, Jamali S, Samadikuchaksaraei A. Imipenem-resistant Pseudomonas aeruginosa strains carry metallo-beta-lactamase gene bla(VIM) in a level I Iranian burn hospital. Burns. 2010;36(6):826-30.

29. Rastegar Lari A, Azimi L, Rahbar M, Fallah F, Alaghehbandan R. Phenotypic detection of Klebsiella pneumoniae carbapenemase among burns patients: first report from Iran. Burns. 2013;39(1):174-6.

30. Khosravi AD, Mihani F. Detection of metallo-beta-lactamase-producing Pseudomonas aeruginosa strains isolated from burn patients in Ahwaz, Iran. Diagn Microbiol Infect Dis. 2008;60(1):125-8.

31. Yousefi S, Farajnia S, Nahaei MR, Akhi MT, Ghotaslou R, Soroush $\mathrm{MH}$, et al. Detection of metallo-beta-lactamase-encoding genes among clinical isolates of Pseudomonas aeruginosa in northwest of Iran. Diagn Microbiol Infect Dis. 2010;68(3):322-5.

32. Karimi A, Rahbar M, Fallah F, Navidinia M, Malekan MA. Detection of integron elements and gene groups encoding ESBLs and their prevalence in Escherichia coli and Klebsiella isolated from urine samples by PCR method. Afr J Microbiol Res. 2012;6(8):1806-9. 